\title{
Training versus service provision: the plight of the core medical training clinic
}

\author{
Authors: William Wareing, ${ }^{*}$ Matthew Tanti, Akriti Naraen and Matthew Grant
}

\author{
Aim \\ To improve access to and availability of clinics for core medical \\ trainees.
}

\begin{abstract}
Methods
Clinics are an invaluable and mandatory part of core medical training (CMT). The difficulties that trainees face in attending clinics was raised at our trust's junior doctor forum. The predominant issue was of limited capacity within rotas to free trainees up to attend clinic while still ensuring adequate ward staffing levels. It was felt that dedicated clinic time incorporated into individual departmental staffing rotas would be more effective in reducing the likelihood of trainees being asked to leave clinics to cover wards.

We proposed the provision of a monthly 'CMT clinic day' for each trainee that would be incorporated into their rota in advance. Agreement for this was obtained from all medical specialty departments. This clinic day could be used for trainees to attend clinics or alternative clinical sessions such as the cardiac catheter suite or endoscopy. A dedicated CMT stream of patients was also opened up in a general medical clinic within the care of the elderly department.

We used a SurveyMonkey questionnaire to assess trainee experiences before and after these interventions and will continue to reassess the strategy's effectiveness through 4-monthly 'plan, do, study, act' cycles.
\end{abstract}

\section{Results}

Of the 13 core medical trainees in the trust, 8 out of 13 completed the pre-intervention survey (survey 1 ) and 9 out of 13 completed the post-intervention survey (survey 2 ).

Following the interventions outlined, $88 \%$ (compared to $37.5 \%$ in survey 1) of trainees reported having clinics incorporated into their specialty rota and the average number of clinics attended per 4-month rotation increased from 4.3 to 5.1 . In survey $2,75 \%$ of trainees felt that the dedicated clinic days were a good idea, with $37 \%$ reporting that it had improved their clinic attendance record. Twenty-five per cent felt that their training had improved as a result of the intervention and $88 \%$ felt that they had adequate senior support when attending clinic.

Despite this improvement only $44 \%$ of trainees felt they would attend the required numbers of clinic for their annual review of competence progression. The main reasons given for this were persistent staffing pressures on wards.

\section{Conclusion}

The intervention has improved both availability and access to clinics for trainees. However, staffing pressures remain a hindrance to training opportunities. Furthermore there was a significant discrepancy in clinic attendance numbers between specialties. The reasons for this are being investigated and addressed with plans to then re-survey the trainees after the next 4-month rotation in order to ensure equity of clinic experience across specialties within CMT.

\section{Conflict of interest statement}

None. 